Laser Chem. 1988, Vol. 8, pp. 377-384

(C) 1988 Harwood Academic Publishers GmbH

Photocopying permitted by license only

Reprints available directly from the Publisher

Printed in the United Kingdom

\title{
Picosecond Studies of Intramolecular Double Proton Transfer by Excite- and Probe- Technique
}

\author{
M. KASCHKE, S. RENTSCH and J. OPFERMANN
}

Department of Physics, Friedrich Schiller University, Jena, Max-Wien-Platz, 6900 JENA, D.D.R.

(Received 3 December, 1987; Accepted 16 February, 1988)

The double proton transfer of $2,2^{\prime}$-bipyridyl-3,3'-diol $\left(\mathrm{BP}(\mathrm{OH})_{2}\right)$ in isooctane after UV excitation has been studied by excite and probe beam spectroscopy with a time resolution of 5 ps. Estimates for the excited state proton transfer rate as well as for the extinction coefficients of the excited state absorption bands of the primarily excited species and of the tautomer have been determined.

KEY WORDS: Intramolecular proton transfer, picosecond spectroscopy.

\section{INTRODUCTION}

Intramolecular proton transfer after excitation by ultrashort light pulses occurs when a strong redistribution of the electron distribution takes place and in subsequence a strong proton affinity in the neighbourhood of a bonded proton arises. Such a transfer process along a short distance of a few $\AA$ is the primary step in many chemical reactions.

Intramolecular proton transfer kinetic studies has been performed e.g. in indigoides, benzoxazoles and methylsalycylates. ${ }^{1,2}$ Cooperative double proton transfer first has been studied in 7-azaindole dimers ${ }^{3}$ and then in $\left[2,2^{\prime}\right.$-bipyridyl $]-3,3^{\prime}$-diol $\left(\mathrm{BP}(\mathrm{OH})_{2}\right){ }^{4,5}$ 
In materials which exhibit proton transfer in the excited singlet state a strongly Stokes shifted fluorescence spectrum will be observed. This flourescence belongs to the state of a tautomer which occurs after the proton transfer, whereas the primary excited state $S_{1}$ shows no fluorescence. For investigation of the proton transfer reaction time resolved measuring methods are suitable: picosecond time resolved streak camera for fluorescence measurements and excite- and probetechnique for transient absorption measurements. (Single photon counting mostly has a too low time resolution.)

The increase of the fluorescence indicate the proton transfer, as well as the absorption kinetics of the primary excited or the secundarely by the chemical reaction occupied states.

\section{CHARACTERISTICS OF BP(OH $)_{2}$}

An insight into the physical characteristics of the molecule under study was taken from. ${ }^{4,5}$ As known, nitrogen atoms become more basic and the hydroxylic oxygen more acidic under excitation to the singlet state in such compounds. This effect provides the driving force for the proton transfer reaction. In $\mathrm{BP}(\mathrm{OH})_{2}$ there is the possibility of a single as well as of a double proton transfer. Single proton transfer results in an uncharged structure with non-zero dipole moment, double proton transfer produces a zwitterionic structure without a resulting dipole moment. The ground state dipole moment of $\mathrm{BP}(\mathrm{OH})_{2}$ is negligibly small.

From spectroscopic studies it is known that both the absorption and the strongly Stokes shifted fluorescence band are not sensitive to solvents. Thus, it can be concluded that the dipole moment upon excitation to the $S_{1}$ state does not change.

The independence of the solvent is one essential argument that double proton transfer in the excited state really takes place. The symmetry of the molecule supports the assumed double proton transfer process.

$\mathrm{BP}(\mathrm{OH})_{2}$ is a strongly planar molecule with a high fluorescence quantum yield of the Stokes shifted band. Related compounds, as BP and $\mathrm{BPOH}$ show only negligible fluorescence. In these compounds rotational modes of the rings and their coupling to solvent modes cause fast radiationless relaxation. In $\mathrm{BP}(\mathrm{OH})_{2}$ the intramolecular $\mathrm{H}$ - 
bonds rigidize the planar structure of the molecule and prevent the ring rotation and the relaxation. The existence of such $\mathrm{H}$-bonds is evident from IR-spectra (See Ref. 4 and cited ref.). The transfer process is nearly independent of temperature and on deuteration. From that a barrierless transfer has been concluded which was estimated to proceed in a sub-picosecond time scale. ${ }^{5}$

\section{EXPERIMENTAL}

The aim of the picosecond spectroscopy is to study the time behaviour of the tautomer fluorescence or an excited state absorption which has to be detected and identified before. The excite- and probespectroscopy allows both possibilities. The picosecond Nd-phosphate glass laser spectrometer used, has the following parameters: ${ }^{6}$ The excitation wavelength are the harmonics of $1054 \mathrm{~nm}$ or the stimulated Raman scattering generated in several materials by these wavelengths. The test radiation is a picosecond continuum generated in heavy water in the spectral range from 430 to $1000 \mathrm{~nm}$.

For excitation of $\mathrm{BP}(\mathrm{OH})_{2}$ we need UV radiation, i.e. the third harmonic of the laser radiation, $351 \mathrm{~nm}$. The exciting pulse has a duration of $5 \mathrm{ps}$ and an energy of about 100 to $200 \mu \mathrm{J}$.

The absorption and flourescence spectra of $\mathrm{BP}(\mathrm{OH})_{2}$ are shown in Figure 1. What we expect after the excitation is to observe an induced flourescence at $500 \mathrm{~nm}$ and to find transient absorption bands either of the primary excited state or of the excited tautomer. From our measurements of intermolecular proton transfer ${ }^{7}$ we know that excited state absorption bands can occur on the short as well as on the long



Figure 1 Absorption (-- ) and fluorescence (-) spectra of $\mathrm{BP}(\mathrm{OH})_{2}$ in isooctane. 
wavelength side of the fluorescence. The bleaching of the ground state absorption cannot be observed because of the limited spectral range of the available test radiation.

Indeed, we found the negative $\Delta D$-values of the fluorescence and, on the short wavelength side, a transient absorption. The $\Delta D$-spectra are a superposition of 25 single excitation events, the $\Delta D$-values amount up to 0.1 .

\section{RESULTS}

The time behaviour of $\mathrm{BP}(\mathrm{OH})_{2}$ was studied in isooctane and in methanol. In both solvents an excited state absorption arises with the exciting pulse, whereas the fluorescence increases with a small delay of about 10 ps to that. The experimental $\Delta D$-curves for isooctane solution are shown in Figure 2 at various delay times.

The transient absorption does not evolve in time in the same way as the fluorescence increases. It must be assumed that transient absorption is a superposition of two spectra, that of the primary excited and that of the tautomeric form of the molecule.

According to the observed delay between induced absorption and fluorescence one must assume that the formation of the tautomeric form requires a few picoseconds to take place. ${ }^{11}$ There are no hints for

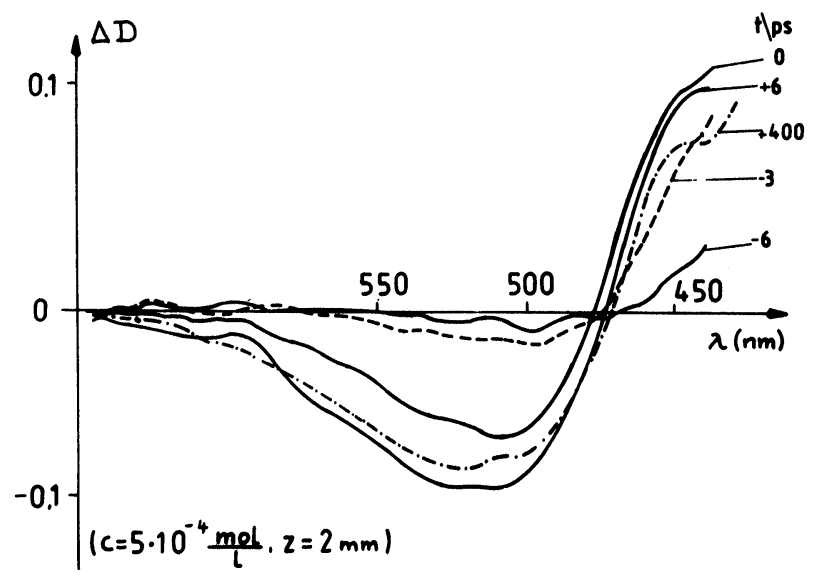

Figure $2 \Delta D$-values of $\mathrm{BP}(\mathrm{OH})_{2}$ in isooctane at various delay times upon excitation. 


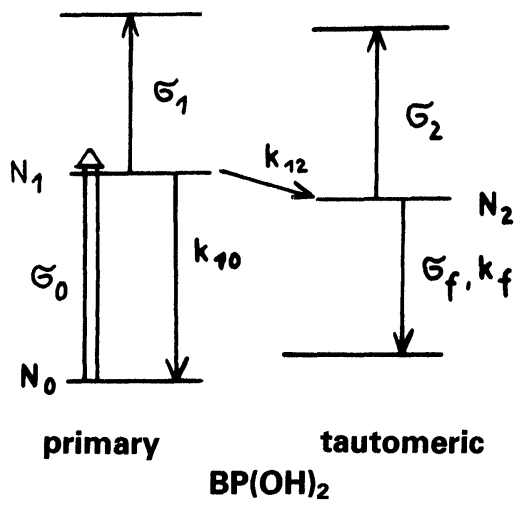

Figure 3 Scheme of the proton transfer.

a stepwise transfer of the two protons of $\mathrm{BP}(\mathrm{OH})_{2}$ in the ps-time resolved spectra. Therefore, we used a simple model which contains the primary and the tautomeric excited states and the corresponding ground states with their absorption and emission to describe our results (see Figure 3). The occupation and depletion of the states will be described with rate equations for the number of excited molecules, normalized to the total number of molecules $N_{t}$ in the irradiated volume (area A, thickness $z, V=0.2 \cdot 10^{-3} \mathrm{~cm}^{-3}$ ). The normalized molecule numbers are $n_{1}=N_{1} / N_{t}$ for the $S_{1}$ state and $n_{2}=N_{2} / N_{t}$ for the excited tautomer. Under consideration of the absorption of the exciting radiation (cross section $\sigma_{0}$ ) holds:

$$
\begin{aligned}
& \frac{d n_{1}}{d t}=N_{p} g(t)\left[1-\exp \left(\sigma_{0} N_{t} n_{1} / A\right)\right]-k_{1} n_{1} \\
& \frac{d n_{2}}{d t}=k_{12} n_{1}-k_{f} n_{2}
\end{aligned}
$$

with the $S_{1}$ depletion rate $K_{1}=k_{10}+k_{12}$, the excited tautomer depletion rate $k_{f}=0.5 \cdot 10^{9} \mathrm{~s}^{8}$ and the photon number density $N_{p}$. For a detailed analysis of the equations the shape of the exciting pulse has to be taken into consideration, the pulse was assumed to be a Gaussian with the line form function $g(t)$ and 5 ps half widths.

The rate equations have been treated by a regulated Gauss-Newton procedure together with a Runge-Kutta subroutine. ${ }^{9,10}$ The experi- 
mentally available values are $\Delta D$-spectra at selected time intervals which contains the excited state absorptions of both states with the cross sections $\sigma_{1}, \sigma_{2}$ and the induced fluorescence with the cross section $\sigma_{f}$ of the tautomer:

$$
D(\lambda, t)=\sigma_{1}(\lambda) N_{1}+N_{2}\left[\sigma_{2}(\lambda)-\sigma_{f}(\lambda)\right]
$$

A possible fluorescence band of the $S_{1}$ has to be located in the UV region outside of the range measured. The emission cross section of the tautomer has been calculated from the spontaneous fluorescence spectrum in arbitrary units $\left(\sigma_{f}=\alpha \sigma_{\mathrm{e}}\right)$. The fluorescence quantum yield has been determined to amount to 0.46 in isooctane. The fitting procedure was applied to the kinetic curves at 14 selected wavelengths within the $\Delta D$-spectra. The computer analysis allows to estimate the unknown rate parameters $k_{1}$ and $k_{12}$ and to determine the unknown cross sections. The calculated kinetics of the state occupation is demonstrated in Figure 4. The $S_{1}$ occupation increases during the exciting pulse, whereas the tautomer excited state will be occupied later. The tautomer kinetics is identical with the temporal develop-

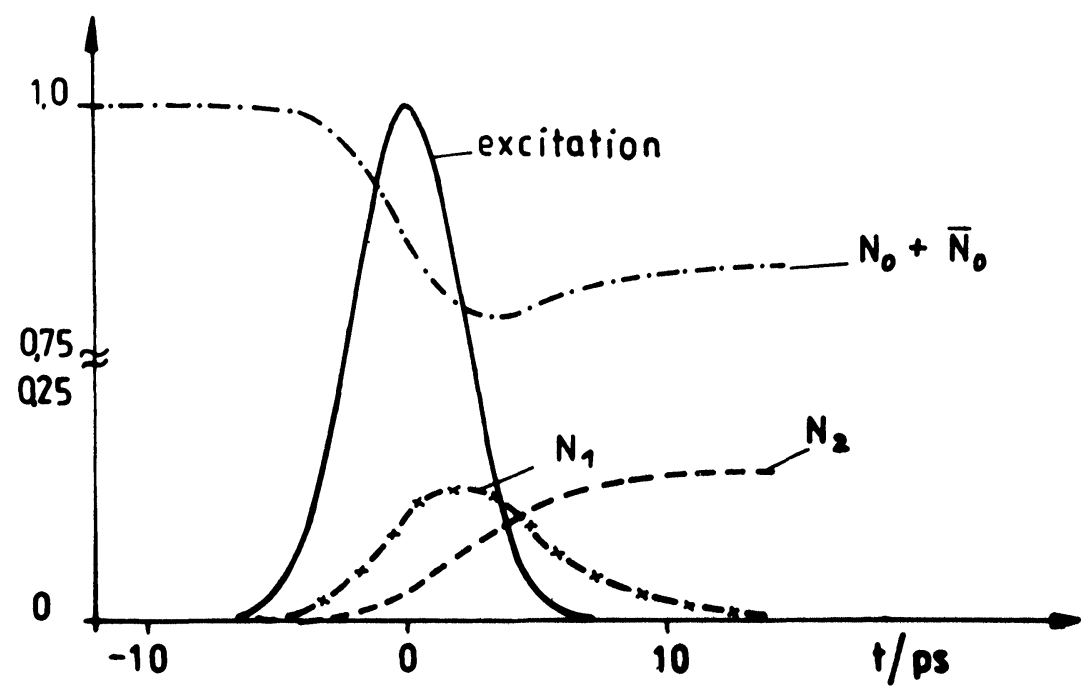

Figure 4 Normalized excited state occupation numbers calculated from the experimental results. 
ment of the induced fluorescence. The rate parameters for $\mathrm{BP}(\mathrm{OH})_{2}$ in isooctane as estimated by the fitting procedure are

$$
\begin{aligned}
k_{1} & =(0.150+0.05) \mathrm{ps}^{-1} \text { and } \\
k_{12} & =(0.147+0.07) \mathrm{ps}^{-1} .
\end{aligned}
$$

The proton transfer process is assumed to be barrierless, but the competing radiationless relaxation of $S_{1}$ is temperature dependent. ${ }^{4,5}$ The maximum absorption cross section of boths excited states amounts to $2 \cdot 10^{-17} \mathrm{~cm}^{2}$. This small cross sections causes the low $\Delta D$-values and limits the accuracy of the measurement $(\Delta D=0.01)$.

In Figure 5 estimates for the absorption coefficients $\varepsilon(\lambda)$ for both the excited states are shown together with the tautomer fluorescence spectrum.



Figure 5 Excited state and excited tautomer absorption spectra, estimated by the fitting procedure and the induced fluorescence spectrum in arbitrary units.

\section{SUMMARY}

The double proton transfer in $\mathrm{BO}(\mathrm{OH})_{2}$ proceeds with $S_{1}$ depletion within $\tau_{g}=3 \mathrm{ps}$. The excited tautomer is formed with the rate 0.147 $\mathrm{ps}^{-1}$. Transient absorption spectra of the two excited states with 
maximum cross section of $2 \cdot 10^{-17} \mathrm{~cm}^{2}$ were found by use of $\mathrm{ps-excite-}$ and probe-spectroscopy and computer analysis of the results.

\section{Acknowledgement}

We thank Dr. H. Bulska and Dr. A. Grabowska for discussions and for the material.

\section{References}

1. F. Doerr in NATO ASI Series C, Vol. 127 Appl. of Ps Spectr. to Chem. ed. by K. B. Eisenthal, 1984, pp. 127-138.

2. P. M. Felker and A. H. Zewail, ibid. pp. 273-291.

3. C. A. Taylor, M. E. El-Bayoni and M. Kasha, Proc. Nat. Acad. Sci. USA 63, 253 (1969).

4. H. Bulska, Chem. Phys. Lett. 98, 308 (1983).

5. H. Bulska, A. Grabowska and Z. H. Grabowski, J. Lum. 35, 186 (1986).

6. T. Damm, M. Kaschke, M. Kresser, F. Noack, S. Rentsch and W. Triebel, Exp. Techn. Phys. 33, 409 (1985).

7. M. Kaschke, J. Kleinschmidt, A. Giraness, Laser Chem. 7, 41 (1987).

8. A. Grabowska, J. Sepiol and H. Bulska, Proc. Intern. Symp. Mol. Lum. Torun 1986, p. 120 .

9. J. Opfermann, Rechentechnik/Datenverarbeitung 22(3), 26 (1985).

10. J. Opfermann, Manual of Software Package "Non-linear parameter estimation" Fr.-Schiller University, 1984 and Software "Kinetics of photoreactions," 1987.

11. The observed delay is somewhat in contrast to steady state fluorescence measurements at low temperatures by H. Bulska (Chem. Phys. Lett. 98, 308 (1983)) and N. P. Ernsting, M. Kaschke (unpublished results of fluorescence measurements in $10 \mathrm{~K}$ argon matrices). The discrepancy is unclear, possibly the solvent introduces a small barrier for the proton transfer. 